\title{
Automated Real-time
} Nucleic Acid Amplification Technology for Rapid and Simultaneous Detection of Tuberculosis and Rifampicin Resistance: Xpert MTB/RIF System Policy Statement 
Policy statement: automated real-time nucleic acid amplification technology for rapid and simultaneous detection of tuberculosis and rifampicin resistance: Xpert MTB/RIF system.

1.Tuberculosis, Multidrug-resistant - diagnosis. 2.Tuberculosis - diagnosis. 3.Rifampin pharmacology. 4.Mycobacterium tuberculosis - isolation and purification. 5.HIV infections diagnosis. 6.Sensitivity and specificity. 7.Guidelines. I. World Health Organization.

ISBN 9789241501545

(NLM classification: WF 310)

\section{(c) World Health Organization 2011}

All rights reserved. Publications of the World Health Organization are available on the WHO web site (www.who.int) or can be purchased from WHO Press, World Health Organization, 20 Avenue Appia, 1211 Geneva 27, Switzerland (tel.: +41 22791 3264; fax: +41 22791 4857; e-mail: bookorders@who.int).

Requests for permission to reproduce or translate WHO publications - whether for sale or for noncommercial distribution - should be addressed to WHO Press through the WHO web site (http://www.who.int/about/licensing/copyright form/en/index.html).

The designations employed and the presentation of the material in this publication do not imply the expression of any opinion whatsoever on the part of the World Health Organization concerning the legal status of any country, territory, city or area or of its authorities, or concerning the delimitation of its frontiers or boundaries. Dotted lines on maps represent approximate border lines for which there may not yet be full agreement.

The mention of specific companies or of certain manufacturers' products does not imply that they are endorsed or recommended by the World Health Organization in preference to others of a similar nature that are not mentioned. Errors and omissions excepted, the names of proprietary products are distinguished by initial capital letters.

All reasonable precautions have been taken by the World Health Organization to verify the information contained in this publication. However, the published material is being distributed without warranty of any kind, either expressed or implied. The responsibility for the interpretation and use of the material lies with the reader. In no event shall the World Health Organization be liable for damages arising from its use. 


\section{Contents}

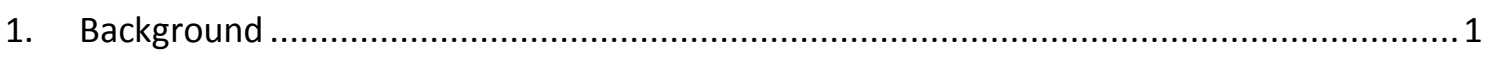

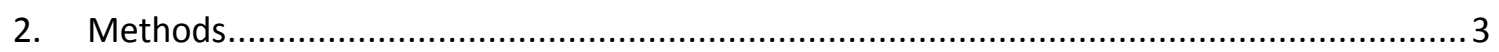

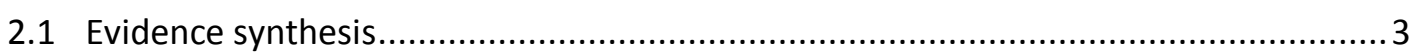

2.2 Decision-making during the Expert Group meeting .........................................4

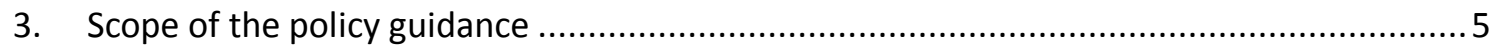

4. Evidence base for policy formulation ........................................................... 5

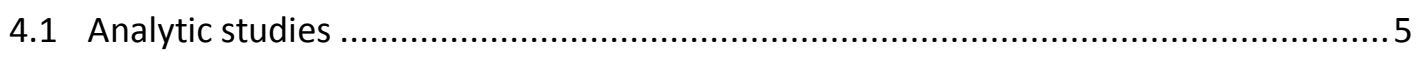

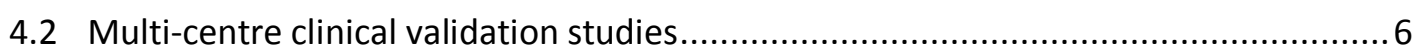

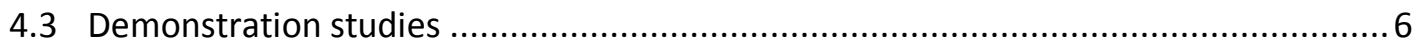

4.4 Unpublished single-centre evaluation studies .............................................8

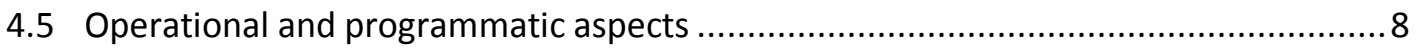

4.6 Cost-effectiveness modeling ................................................................... 9

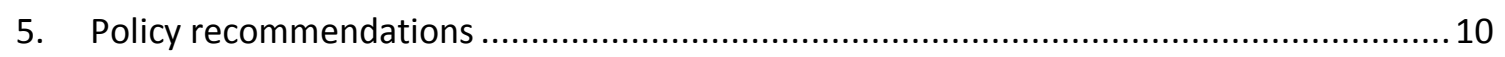

6. Implementation considerations ............................................................... 11

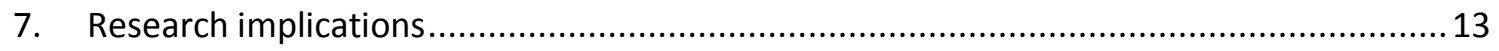

8. Plans for supporting roll-out of Xpert MTB/RIF ................................................ 13

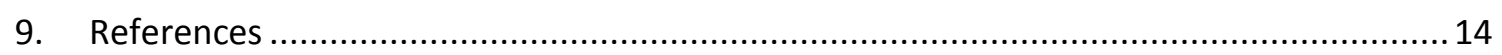

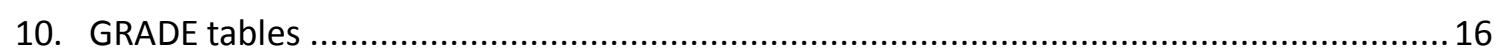

Table 1. GRADE evidence profile: Xpert MTB/RIF as a replacement test for conventional culture and DST in FIND- coordinated multi-centre evaluation and demonstration studies

Table 2. GRADE summary of findings - Diagnostic accuracy of the Xpert MTB/RIF assay in multi-centre clinical validation studies

Table 3. GRADE summary of findings - Diagnostic accuracy of the Xpert MTB/RIF assay in multi-centre demonstration studies.

Table 4. GRADE summary of findings - Diagnostic accuracy of the Xpert MTB/RIF assay in single-centre unpublished studies.

11. Annexes 


\section{EXECUTIVE SUMMARY}

\section{Background}

Earlier and improved tuberculosis (TB) case detection - including smear-negative disease, often associated with HIV co-infection - as well as expanded capacity to diagnose multidrug-resistant tuberculosis (MDR-TB) are global priorities for TB control. Conventional laboratory methods are slow and cumbersome and novel technologies for rapid detection are therefore the focus of TB research and development. With support from $\mathrm{NIH}$, the Foundation for Innovative New Diagnostics (FIND) has partnered with Cepheid, Inc. (Sunnyvale, CA) and the University of Medicine and Dentistry of New Jersey (UMDNJ, Newark, NY) to develop a TB-specific automated, cartridge-based nucleic amplification assay (Xpert MTB/RIF) based on the GeneXpert multi-disease platform, currently unique in its simplification of molecular testing with fully integrated and automated sample preparation, amplification and detection required for real-time polymerase chain reaction. Xpert MTB/RIF detects $M$. tuberculosis as well as rifampicin resistance-conferring mutations directly from sputum, in an assay providing results within two hours. Data from published papers, large multi-centre laboratory validation and demonstration studies coordinated by FIND, and unpublished data investigator-driven, single-centre studies were recently reviewed by WHO with the aim to develop policy guidance on Xpert MTB/RIF use.

\section{Summary of results}

Results from analytical studies showed that the Xpert MTB/RIF assay has analytic sensitivity of five genome copies of purified DNA, and $131 \mathrm{cfu} / \mathrm{ml}$ of $M$. tuberculosis spiked into sputum. The molecular beacons which target the $r p o B$ gene cover all the mutations found in $>99.5 \%$ of all rifampicin resistant strains. There is no cross-reactivity with non-tuberculous mycobacteria, and TB and rifampicin resistance were correctly detected in the presence of non-tuberculous DNA or mixed susceptible and resistant strains. The sample reagent added in a 2:1 ratio to sputum was shown to kill $>6 \log _{10} \mathrm{cfu} / \mathrm{ml}$ of $M$. tuberculosis with 15 minutes of exposure, and to render $>97 \%$ of smear-positive samples negative by $\amalg$ culture. The Xpert inoculation procedure and sample testing generated no detectable infectious aerosols.

Results from controlled clinical validation trials involving 1,730 individuals suspected of TB or MDR-TB prospectively enrolled in four distinctly diverse settings showed that $92.2 \%$ of culturepositive patients were detected by a single direct Xpert MTB/RIF test. Sensitivity of a single Xpert MTB/RIF test in smear-negative/culture-positive patients was $72.5 \%$ and increased to $90.2 \%$ when three samples were tested. Xpert MTB/RIF specificity was $99 \%$. Xpert MTB/RIF detected rifampicin resistance with $99.1 \%$ sensitivity and excluded resistance with $100 \%$ specificity.

Results from field demonstration studies involving 6,673 individuals prospectively enrolled in six distinctly different settings confirmed these findings:

Test accuracy was retained, with a single Xpert MTB/RIF test directly from sputum detecting $99 \%$ of smear-positive patients and $>80 \%$ of patients with smear-negative disease. HIV co-infection substantially decreased the sensitivity of microscopy, but did not significantly affect Xpert MTB/RIF performance. Rifampicin resistance was detected with $95.1 \%$ sensitivity and $98.4 \%$ specificity.

Mean time to detection was $<1$ day for Xpert MTB/RIF, 1 day for microscopy, 17 days for liquid culture and $>30$ days for solid culture. Rifampicin resistance was detected in $<1$ day with Xpert MTB/RIF vs an average of 75 days for phenotypic DST. When Xpert MTB/RIF results were not used to direct therapy, smear-negative TB patients started treatment after a median period of 58 days, compared to a median of 4 days when Xpert MTB/RIF results were used.

Operational aspects assessed confirmed robustness of Xpert MTB/RIF under varying temperature and humidity conditions, minimal training required of personnel, and high levels of user satisfaction. Storage of cartridges in high-volume settings was a concern given lack of adequate space. Waste generated was considerable more than for microscopy. Xpert MTB/RIF requires 
uninterrupted and stable electrical power supply and annual validation of the system, which may pose a problem in rural/remote settings.

The negative predictive value (NPV') of Xpert MTB/RIF assay is over $99 \%$ in settings with both low and high prevalence of rifampicin resistance, i.e. a negative result accurately excludes the possibility for rifampicin resistance.

The positive predictive value (PPV) for rifampicin resistance using Xpert MTB/RIF depends on underlying prevalence of rifampicin resistance. In settings or patient groups where rifampicin resistance is rare, the PPV of Xpert MTB/RIF testing is adversely affected. The PPV of Xpert MTB/RIF can be greatly improved by careful risk assessment in individual patients and targeted Xpert MTB/RIF testing.

Cost-effectiveness modeling indicated that the use of Xpert MTB-RIF significantly increased TB case-finding (by roughly 30\%) when used as a replacement or add-on test to microscopy. Use of Xpert MTB/RIF as replacement for conventional culture and DST also significantly increased MDR case-finding (roughly three-fold). The capital and running costs of Xpert MTB/RIF are substantially greater than those of microscopy, though similar to the cost for performing culture and drug susceptibility testing.

Results from single-centre evaluation studies with varying design and study populations reported sensitivity in detecting TB ranging from $70 \%$ to $100 \%$ in culture-positive patients and around $60 \%$ in those with smear-negative disease. Specificity ranged from $91 \%$ to $100 \%$. Pooled crude sensitivity for TB detection was $92.5 \%$ and pooled crude specificity was $98 \%$. Average rifampicin sensitivity and specificity were around $98 \%$ and $99 \%$ respectively.

\section{Conclusions}

The WHO evidence synthesis process confirmed a solid evidence base to support widespread use of Xpert MTB-RIF for detection of TB and rifampicin resistance. It is therefore recommended that:

1) Xpert MTB/RIF should be used as the initial diagnostic test in individuals suspected of MDR-TB or HIV-associated TB (strong recommendation);

2) Xpert MTB/RIF may be used as a follow-on test to microscopy in settings where MDR and/or HIV is of lesser concern, especially in smear-negative specimens (conditional recommendation, recognising major resource implications).

Xpert MTB/RIF technology does, however, not eliminate the need for conventional microscopy culture and DST, which are required to monitor treatment progress and to detect resistance to drugs other than rifampicin. In settings or patient groups where rifampicin resistance is rare, Xpert MTB/RIF results indicating rifampicin resistance should be confirmed by conventional DST or LPA.

In addition, several operational conditions need to be met for successful implementation of Xpert MTB/RIF - stable electrical supply, a maximum ambient operating temperature of $30^{\circ} \mathrm{C}$ for the GeneXpert device, security against theft, dedicated trained personnel, adequate storage space, annual calibration of the instrument by a commercial supplier, and biosafety precautions similar to those for direct sputum microscopy should all be in place.

A Global Consultation called by WHO in December 2010 discussed the implementation considerations for scale-up of Xpert MTB/RIF and achieved broad consensus on the way forward. Key outcomes of the consultation were agreement on interim diagnostic algorithms, the positioning of Xpert MTB/RIF in risk groups at different levels of health services, and

\footnotetext{
${ }^{i}$ NPV for rifampicin resistance: the proportion of diagnosed rifampicin-susceptible cases that are truly susceptible ii PPV for rifampicin resistance: the proportion of diagnosed rifampicin-resistant cases that are truly resistant
} 
implementation considerations for programmatic roll-out of Xpert MTB/RIF to optimize use and benefits of the technology.

Finally, a key consideration is the need for rapid access to appropriate treatment and care for all TB and MDR-TB patients who will be identified by the introduction of Xpert MTB/RIF. Implementation of Xpert MTB/RIF technology should therefore be phased in within the context of comprehensive national TB and MDR-TB strategic plans. 


\section{Acknowledgements}

This document was prepared by Karin Weyer, Christopher Gilpin and Fuad Mirzayev (WHO Stop TB Department) on the basis of consensus at an international Expert Group Meeting convened by WHO in Geneva on $2^{\text {nd }}$ September 2010.

WHO gratefully acknowledges the contributions of the Chair of the Expert Group (Holger Schünemann) and the members of the Expert Group (Annex 1) who developed the recommendations.

The findings and recommendations from the Expert Group Meeting were presented to the WHO Strategic and Technical Advisory Group for Tuberculosis (STAG-TB, Annex 2) in September 2010. STAG-TB acknowledged the transforming potential of this new technology and the solid evidence base to support its widespread use for detection of TB and rifampicin resistance.

A Global Consultation was called by WHO on 30 November - 2 December 2010 where implementation considerations for scale-up of Xpert MTB/RIF were discussed and broad consensus on the way forward was achieved. In addition to WHO regional and headquarters staff 59 participants were included in the consultation and included representatives from 14 National TB Programmes (Benin, Brazil, China, Georgia, Indonesia, Kenya, Moldova, Nigeria, Pakistan, Peru, Senegal, South Africa, Ukraine, Viet Nam) and heads of five TB Supranational Reference Laboratories. Other participants represented donor agencies, researchers, clinicians, epidemiologists, and community representatives.

This document was finalized following consideration of all comments and suggestions from the participants of the Expert Group, STAG-TB and the Global Consultation.

Funding by USAID is gratefully acknowledged, through USAID-WHO Consolidated Grant No. GHA-G00-09-00003.

\section{Declarations of Interest}

Expert Group members were asked to submit completed Declaration of Interest (DOI) forms. These were reviewed by the WHO Legal Department prior to the Expert Group meeting. DOI statements were summarised by the co-chair of the Expert Group meeting (Karin Weyer, WHO-STB) at the start of the meeting.

Three expert group members (Richard Anthony, Daniela Cirillo, Doris Hillemann) declared FIND (Foundation for Innovative New Diagnostics) support for unrelated research activities. These declarations were deemed to be insignificant.

Frank Cobelens declared contractual agreements with FIND to conduct cost-effectiveness studies of the Xpert MTB/RIF assay as well as a BMGF consultancy to conduct an impact assessment of the Xpert MTB/RIF. This was deemed to be significant. Therefore, Frank Cobelens was present as observer to the meeting, did not participate in the GRADE evaluation process, and did not contribute to the meeting discussions where recommendations were developed.

FIND participants provided technical clarifications and were present as observers only to the sessions where technical and operational performance of the Xpert MTB/RIF assay was discussed. They were asked to recuse themselves once the meeting proceeded to sessions on GRADE evaluation and recommendations. Neither FIND participants nor other observers provided comments on the final document. 


\section{AUTOMATED REAL-TIME NUCLEIC ACID AMPLIFICATION TECHNOLOGY FOR RAPID AND SIMULTANEOUS DETECTION OF TUBERCULOSIS AND RIFAMPICIN RESISTANCE: Xpert MTB/RIF SYSTEM}

\section{Background}

Earlier and improved tuberculosis (TB) case detection - including smear-negative disease - as well as expanded capacity to diagnose multidrug-resistant tuberculosis (MDR-TB) are global priorities for TB control. MDR-TB poses formidable challenges due to its complex diagnostic and treatment requirements, while HIV-associated TB largely goes undetected due to the limitations of current diagnostic techniques. Alarming increases in MDR-TB, the global emergence of extensively drugresistant TB (XDR-TB), documented institutional transmission, and rapid mortality in MDR-TB and XDR-TB patients with HIV co-infection have highlighted the urgency for rapid screening methods.

Conventional methods for mycobacteriological culture and drug susceptibility testing (DST) are slow and cumbersome, requiring sequential procedures for isolation of mycobacteria from clinical specimens, identification of Mycobacterium tuberculosis complex, and in vitro testing of strain susceptibility to anti-TB drugs. During this time patients may be inappropriately treated, drugresistant strains may continue to spread, and amplification of resistance may occur. Novel technologies for rapid detection of anti-TB drug resistance have therefore become a priority in TB research and development.

Since the development in the early 1980s of the polymerase chain reaction (PCR) - the first and most familiar method to amplify nucleic acid sequences - molecular diagnostics have been widely expected to have a major impact on clinical medicine. However, despite several theoretical advantages, the use of molecular tests for TB has been limited, largely due to the complexities of DNA extraction, amplification and detection, and the biosafety concerns related to manipulating $M$. tuberculosis organisms. In addition, commercial nucleic acid amplification tests (NAAT) proved to be significantly less sensitive than microbiological culture, especially for smear-negative TB. Moreover, culture largely remained necessary as a precursor to drug-susceptibility testing, while scale-up of conventional culture and drug-susceptibility services remained slow and expensive, compounded by huge demands on laboratory infrastructure and human resources.

Over the past five years, and with support from NIH, the Foundation for Innovative New Diagnostics (FIND) has partnered with Cepheid, Inc. (Sunnyvale, CA) and the University of Medicine and Dentistry of New Jersey (UMDNJ, Newark, NY) to develop an automated, cartridge-based NAAT for TB based on the GeneXpert multi-disease platform. The GeneXpert system was launched in 2004 and simplifies molecular testing by fully integrating and automating the three processes (sample preparation, amplification and detection) required for real-time PCR-based molecular testing. The GeneXpert platform is currently the only one of its kind and uses a cartridge containing lyophilised reagents, buffers and washes. Target detection and characterization is performed in real time using a sixcolour laser detection device.

The Xpert MTB/RIF system is a recently developed TB-specific application, designed for the GeneXpert platform, to detect $M$. tuberculosis as well as rifampicin resistance-conferring mutations directly from sputum, in an assay providing results within two hours. Testing involves three manual steps: the addition of sample treatment reagent to liquefy and inactivate the bacteria in the sputum, transfer of $2 \mathrm{ml}$ of liquefied sputum into the cartridge, and loading of the cartridge into the device for the assay. All further steps are automated. The assay can be performed directly on a clinical sputum 
specimen or on a decontaminated concentrated sputum pellet. Testing procedures are outlined below:

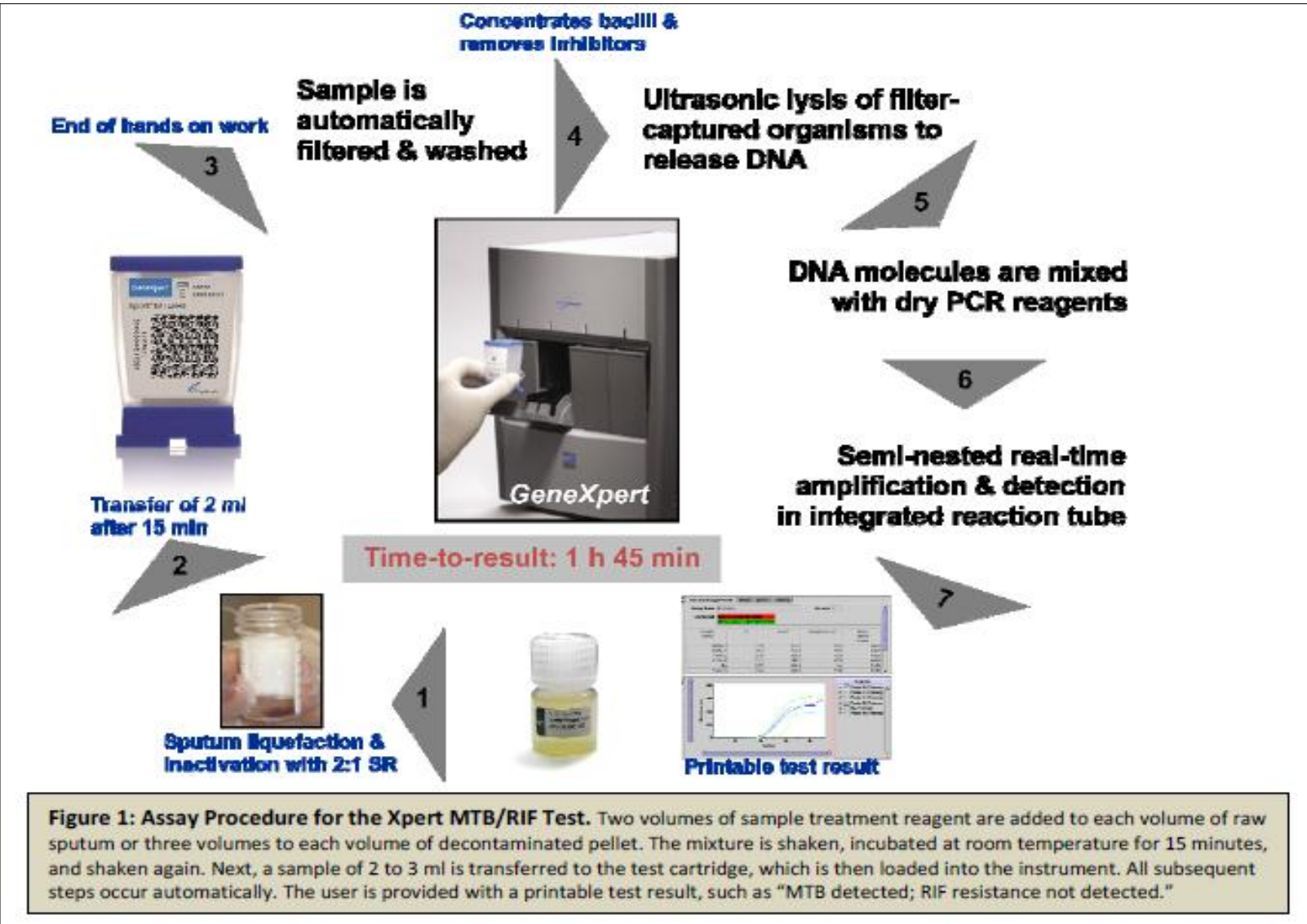

The Xpert MTB/RIF assay is based on hemi-nested real-time PCR amplifying the rpoB gene target. Molecular beacons using novel fluorophors and quenchers are used to detect hybridization to each of the five amplified target regions of the gene. Bacillus globigii, a spore-forming soil organism, is used as a full process control, acting as quality check for bacterial trapping, bacterial lysis, DNA extraction, amplification, and probe detection.

The Xpert MTB/RIF assay has been designed to be robust and easy to use. Xpert MTB/RIF cartridges and the specimen reagent should be stored at $2-28^{\circ} \mathrm{C}$ as per the manufacturer's recommendations. The cartridges are pre-loaded with all reagents necessary for sample processing, DNA extraction, amplification, and laser detection of target amplicon binding to the molecular beacons. The ambient operating temperature for the GeneXpert instrument is currently limited to a maximum of $30^{\circ} \mathrm{C}$.

The assay was designed to be safe to use in microscopy laboratories where biological safety cabinets are usually not available. The sample treatment reagent is mycobactericidal, rapidly killing $M$. tuberculosis during the liquefaction process, and studies have confirmed that no aerosolation of mycobacteria occur while running the device.

GeneXpert instruments with varying capacities are available. The instruments assessed in the studies described below are four-module devices with capacity of running a maximum of 20 tests per day.

Both the GeneXpert platform and the Xpert MTB/RIF assay are under multiple patents. The GeneXpert platform is FDA approved. The assay is manufactured under ISO 13485:2003 certification and approved by the Regulatory Authority in Europe (CE-Marked), with FDA approval pending. 
The GeneXpert platform and the Xpert MTB/RIF assay are currently the only mature technology representing a new generation of automated molecular diagnostic platforms. Others are at prototype stage and expected to become available in due course. These will need to be subjected to the same level of validation and expert review before their implementation can be recommended by WHO.

\section{Methods}

\subsection{Evidence synthesis}

In September 2010, an Expert Group was convened by the Stop TB Department of the World Health Organization (WHO) to assess all available data on Xpert MTB/RIF with a view towards policy recommendations on its use. Data from four published papers, large multi-centre laboratory validation and demonstration studies coordinated by FIND, and unpublished data from 12 investigator-driven, single-centre studies were made available to WHO to assess assay performance, the feasibility of programmatic implementation, and issues to be addressed in future research.

In accordance with current WHO standards for evidence assessment in the formulation of policy recommendations, the GRADE system ${ }^{1}$ (http://www.gradeworkinggroup.org) was used for the evidence synthesis process, providing a systematic, structured framework for evaluating both the accuracy and the patient/public health impact of new interventions.

The following review questions were pre-defined in consultation with the WHO Expert Group:

1. What is the overall diagnostic accuracy of Xpert MTB/RIF for the diagnosis of TB and rifampicin resistance?

2. What is the diagnostic accuracy of Xpert MTB/RIF for the diagnosis of smear-negative TB?

GRADE evidence profiles were prepared to assess the diagnostic accuracy of the Xpert MTB/RIF for the detection of pulmonary TB and the detection of rifampicin resistance compared with conventional culture and DST as reference tests. Using the GRADE framework, sensitivity and specificity results were interpreted as proxy measures for patient-important outcomes based on the relative importance/impact of false-positive and false-negative results: Poor sensitivity would result in false-negative results where TB and MDR-TB patients would be missed with negative consequences for morbidity, mortality and transmission of disease. Poor specificity would result in false-positive results where patients without TB or MDR-TB would be prescribed unnecessary treatment, with negative consequences such as serious adverse events related to second-line anti-TB drug use.

For each outcome, the GRADE quality of evidence was initially regarded as 'high' since all studies were cross-sectional or cohort studies, prospectively enrolling patients suspected with TB and/or MDR-TB. The quality of evidence and study limitations were also assessed using six GRADE criteria: (1) study design; (2) risk of bias; (3) directness; (4) inconsistency; (5) imprecision; and (6) publication/reporting bias. It was noted that the majority of studies did not enroll children and that studies were conducted in largely urban settings due to the need for quality-assured culture and DST as reference methods. As the intended use of the Xpert MTB/RIF test is at district and sub-district levels, the quality of evidence was downgraded by one point to 'moderate'.

The system used to establish the strength and ranking of the recommendations involved assessing and balancing each question according to: (1) desirable and undesirable effects; (2) quality of available evidence; (3) values and preferences related to intervention in different settings; and (4) cost considerations for different epidemiological settings. 
The findings and recommendations from the Expert Group Meeting were presented to the WHO Strategic and Technical Advisory Group for Tuberculosis (STAG-TB) in September 2010. STAG-TB acknowledged the transforming potential of this new technology and the solid evidence base to support its widespread use for detection of TB and rifampicin resistance ${ }^{2}$. STAG-TB also acknowledged the need for urgent access to this innovation in individuals at risk of MDR-TB and HIVassociated TB in resource-constrained settings. STAG-TB subsequently recommended that WHO:

1. Proceed with detailed policy guidance on the use of Xpert MTB/RIF;

2. Develop a global strategy for rapid uptake of Xpert MTB/RIF in a systematic and phased approach, including mechanisms to monitor and assess the roll-out of Xpert MTB/RIF, with a clear plan to document the impact on case detection, MDR-TB response, scale-up and costeffectiveness;

3. Proceed with a Global Consultation on the implementation considerations for scale-up of Xpert MTB/RIF under routine programme conditions (including diagnostic algorithms, logistics, procurement and distribution, quality assurance, and waste disposal);

4. Assist countries with technical support and planning for inclusion of Xpert MTB/RIF in revised diagnostic algorithms.

\subsection{Decision-making during the Expert Group meeting}

The Expert Group meeting was co-chaired by the STB secretariat and a GRADE evidence synthesis expert. Decisions were based on consensus (preferred option) and by voting (with simple majority rule) if consensus was not achieved. Concerns and opinions by Expert Group members were noted and included in the final meeting report ${ }^{3}$. The detailed meeting report was prepared by the STB secretariat and underwent several iterations (managed by the STB secretariat) before final sign-off by Expert Group members.

Draft WHO policy guidance based on the consensus recommendations was subsequently prepared by the STB secretariat and presented to STAG-TB. Final policy guidance was circulated to the Expert Group and STAG-TB members following a similar iterative process as described above.

The final policy guidance document was approved by the WHO Guidelines Review Committee (GRC), having satisfied the GRC requirements for guideline development.ii

iiiGRC statement: This guideline was developed in compliance with the process for evidence gathering, assessment and formulation of recommendations, as outlined in the WHO Handbook for Guideline Development (current version). 


\section{Scope of the policy guidance}

This document provides a pragmatic summary of the evidence and recommendations related to Xpert MTB/RIF, and should be read in conjunction with the detailed findings from the Xpert MTB/RIF Expert Group Meeting Report 2013 ${ }^{3}$, the WHO Framework for Implementing TB Diagnostics, $2010^{4}$ and the WHO Rapid Implementation of Xpert MTB/RIF, 2011 document $^{5}$. These documents are available on http://www.who.int/tb/dots/laboratory/policy/en and provide guidance on the implementation of currently approved WHO diagnostic tools and methods, within the local context of country infrastructure, available resources, TB/MDR-TB epidemiology, and TB policy reform.

None of the existing TB diagnostic tools are mutually exclusive and they can be implemented in various combinations in country screening and diagnostic algorithms, which are highly setting and resource specific. Expert laboratory input is therefore needed to define the most cost-effective and efficient algorithms in individual countries, guided by WHO standards and procedures, and within a framework of overall, integrated laboratory strengthening activities.

This policy guidance should be used to support implementation of Xpert MTB/RIF technology for TB diagnosis and rifampicin resistance detection within National TB Programmes (NTPs), and is intended to be used by NTB Managers and Laboratory Directors, in coordination with external laboratory consultants, donor agencies, technical advisors, laboratory technicians, laboratory equipment procurement officers, warehouse managers, private-sector service providers, other relevant government sectors, and implementing partners involved in country-level TB laboratory strengthening. Individuals responsible for programme planning, budgeting, resource mobilization, and training activities for TB diagnostic services may also benefit from using this document.

Review of additional data from implementation sites will be done annually, with ongoing refinement of the policy guidance based on more extensive field evaluation of the new technology after implementation, including country-specific cost-effectiveness and cost-benefit analyses.

Date of review: 2015

\section{Evidence base for policy formulation}

\subsection{Analytic studies}

Limits of detection (LOD) studies using bacterial genomic DNA and whole organisms spiked into buffer or uninfected sputum showed the Xpert MTB/RIF assay to have an analytic sensitivity of five genome copies of purified DNA and $131 \mathrm{cfu} / \mathrm{ml}$ of $M$. tuberculosis spiked into sputum. The specificity of the assay, tested with a large variety of non-tuberculous mycobacteria, fungi and viruses, showed the assay to correctly exclude all non-tuberculous isolates.

The molecular beacons were designed based on published and experimental data covering 4,115 mutations in the RRDR region from 4,072 strains of $M$. tuberculosis and covered the mutations found in $>99.5 \%$ of all rifampicin resistant strains. TB and rifampicin resistance were correctly detected even in the presence of DNA from non-tuberculous mycobacteria or mixed susceptible and resistant strains.

Two important biosafety features were confirmed: First, the sample reagent added in a 2:1 ratio to sputum was shown to kill $10^{7} \mathrm{log} \mathrm{cfu} / \mathrm{ml}$ of $M$. tuberculosis with 15 minutes of exposure at room temperature and to render $>97 \%$ of smear-positive specimens negative by conventional culture. 
Second, aerosol studies while running the device spiked with high concentrations of mycobacteria did not detect any infectious aerosols generated.

\subsection{Multi-centre clinical validation studies}

Controlled clinical trials were conducted by FIND in four geographically diverse settings with varying underlying TB, HIV and MDR-TB prevalence (Azerbaijan, India, Peru, South Africa), prospectively evaluating 1,730 individuals suspected of either drug-susceptible or MDR-TB. A total of 4,386 sputum specimens from 1,462 eligible patients were tested by Xpert MTB/RIF as well as microscopy and two conventional culture methods.

\section{Detection of pulmonary TB}

The proportion of culture-positive patients detected with a single, direct Xpert MTB/RIF test was $92.2 \%$ vs. $96.0 \%$ for testing two specimens (one direct and one pellet from a concentrated specimen) and $97.6 \%$ for testing three (one direct plus two pellets). No significant difference was observed between tests on untreated sputum and those on decontaminated pellets.

In smear-negative, culture-positive patients, the addition of a second Xpert MTB/RIF test increased sensitivity by $12.6 \%$ from $72.5 \%$ to $85.1 \%$, and a third test further increased sensitivity by $5.1 \%$ to $90.2 \%$. There was no significant variation in sensitivity across sites. A single, direct Xpert MTB/RIF test identified a greater proportion of culture-positive patients than did a single $\amalg$ culture.

HIV status was known for 976 (40.2\%) of patients. Among 392 HIV-positive patients with pulmonary TB, the sensitivity of Xpert MTB/RIF was $93.9 \%$, compared to $98.4 \%$ in HIV-negative patients.

Among 105 patients with culture-negative results treated for TB based on clinical symptoms and showing improvement, $29.3 \%$ had a positive Xpert MTB/RIF result.

The specificity of Xpert MTB/RIF, confirmed by clinical and microbiological follow-up of TB suspects, was $99 \%$.

\section{Detection of rifampicin resistance}

Rifampicin resistance is a reliable proxy for MDR-TB in most settings, and the Xpert MTB/RIF technology probes for five beacons in the $r p o B$ gene comprising the vast majority of rifampicin resistance-conferring mutations. Xpert MTB/RIF correctly detected rifampicin resistance in 209 of 211 patients (99.1\% sensitivity) and in all 506 patients with rifampicin susceptibility (100\% specificity), when compared against phenotypic testing and sequencing results to resolve 15 discordant results. Overall, $97.6 \%$ (200/205) of rifampicin resistant cases were confirmed to have multi-drug resistance on phenotypic testing.

\section{Indeterminate results}

The rate of indeterminate Xpert MTB/RIF results was 3.7\%, lower than the rate of culture contamination. Valid results were obtained in $92.8 \%$ in which enough material remained for the test to be repeated.

\subsection{Demonstration studies}

Demonstration studies coordinated by FIND involved 6,673 mostly adult subjects presenting with symptoms suggestive of pulmonary TB from nine sites in Azerbaijan, India, Peru, the Philippines, South Africa and Uganda. The patient populations were very diverse, with prevalence of HIV ranging from $<1 \%$ to $100 \%$ and MDR prevalence ranging from $2 \%$ to $54 \%$. 
Given the need for reference standards, access to quality-assured conventional culture and DST was pre-requisite and participating centres were therefore largely based in urban or peri-urban areas. All sites categorized patients into MDR risk patients (previously treated, non-converting patients, treatment failures, symptomatic contacts of confirmed MDR cases) and TB case detection patients (others).

Patients were divided into four categories for analysis: smear-positive/culture-positive; smearnegative/culture-positive; smear-negative/culture-negative without treatment (no TB); and smearnegative/culture-negative with treatment based on clinical and radiological findings (clinical TB).

\section{Detection of pulmonary TB}

High sensitivity of Xpert MTB/RIF was confirmed in these studies, with a single test directly on sputum detecting $99 \%$ of smear-positive/culture-positive patients and $>80 \%$ of patients with smearnegative disease. In comparison, a single, direct smear performed on the same sputum specimen as Xpert MTB/RIF had a sensitivity in culture-positive cases of 59.5\% $(95 \mathrm{Cl} 56 \%-63 \%)$. There was no significant difference in specificity (99\%; 95Cl 98\% - 99\%).

A single, direct Xpert MTB/RIF test (sensitivity $90.8 \%$; $95 \mathrm{Cl} 88 \%$ - 93\%) identified a similar proportion of overall culture-positive patients than did a single $\mathrm{L}$ culture (sensitivity $89.8 \% ; 95 \mathrm{Cl} 87 \%-92 \%$ ).

Among HIV-positive patients with culture-confirmed pulmonary TB, the sensitivity of Xpert MTB/RIF was $86 \%$ compared with $92 \%$ in HIV-negative patients. HIV co-infection did not significantly affect Xpert MTB/RIF performance to detect smear-negative/culture-positive patients; in comparison, the sensitivity of smear microscopy was significantly reduced (to $47 \%$ compared to $65 \%$ in HIV-negative patients).

Among 120 TB suspects who were culture-negative but were treated for TB on the basis of clinical symptoms and improved on TB treatment, $19.2 \%$ had positive Xpert MTB/RIF results.

\section{Detection of rifampicin resistance}

Rifampicin resistance is determined by the Xpert MTB/RIF software on the basis of late or absent probe signals. The sensitivity and specificity for detecting rifampicin resistance using the initial software was $98.4 \%$ and $96.1 \%$ respectively. During the early phase of the Field Demonstration studies two false-positive rifampicin resistance calls by Xpert MTB/RIF were reported from South Africa. These two cases were found to be susceptible by line probe assay (used as reference standard) and the absence of mutations was confirmed by sequencing and susceptibility confirmed by phenotypic DST.

A root cause analysis showed that the false calls resulted from some of the molecular beacons generating their signal slightly later than the limit imposed by the software. A review of test results from other sites identified a small number of similar looking artifacts.

Since assay specificity for rifampicin resistance was considered crucial, especially for low MDR prevalence settings, the decision was made to implement a software change for all settings. With these changes, sensitivity for rifampicin detection was slightly reduced to $95.1 \%$ and specificity increased to $98.4 \%$.

Rifampicin mono-resistance was uncommon (1.4\%) and the overall predictive values for MDR using rifampicin resistance detection were high (positive predictive value $92.9 \%$; negative predictive value 98.9\%). There were no reported amplicon contamination events.

\section{Indeterminate results}

Indeterminate Xpert MTB/RIF results were low at $2.5 \%$ and repeated testing led to a valid result in $97 \%$ of patients. In comparison, the overall contamination rate for culture was $4.7 \%$. 


\section{Time to detection of TB and rifampicin resistance}

The mean times to TB detection were $<1$ day for Xpert MTB/RIF, 1 day for microscopy, 17 days for liquid culture and $>30$ days for solid culture. Rifampicin resistance was detected in $<1$ day with Xpert MTB/RIF vs an average of 75 days with conventional phenotypic testing.

\section{Time to treatment initiation}

The impact of speed on utility of results was documented in the Field Demonstration studies: $0.5 \%$ of Xpert MTB/RIF results were not reported to the health services, compared to $1.9 \%$ of microscopy results, $14 \%$ of culture results and $20 \%$ of conventional DST results.

During the validation phase when Xpert MTB/RIF was not used to direct therapy, smear-negative TB patients started treatment after a median of 58 days; during the implementation phase when Xpert MTB/RIF was used to direct treatment, the median time to initiation fell to 4 days.

\subsection{Unpublished single-centre evaluation studies}

Unpublished single-centre evaluation studies yielded similar performance results on 4,575 tests with the Xpert MTB/RIF assay, with reported sensitivity in detecting TB ranging from $70 \%$ to $100 \%$ in culture-positive patients and around $60 \%$ in those with smear-negative disease. Specificity ranged from $91 \%$ to $100 \%$. Pooled crude sensitivity for all Xpert MTB/RIF tests performed was $92.2 \%$ and pooled crude specificity was $98 \%$. Average rifampicin sensitivity and specificity were around $98 \%$ and $99 \%$ respectively.

\subsection{Operational and programmatic aspects}

\section{Robustness}

Technical problems over a one-year period (11 instruments and 44 modules) included module failure (one site, one module), module not detected (two sites, one module each), cartridge stuck inside the module (one site, one module), error message due to high temperature (one site), and error in printout of results (three sites). All technical problems were resolved quickly, mostly with remote support.

Operating temperatures routinely recorded by the GeneXpert instrument showed that the maximum operating temperature recommended by the manufacturer $\left(30^{\circ} \mathrm{C}\right)$ was exceeded in several sites, without adversely affecting instrument or test performance. However, all GeneXpert instruments were new and long-term data on the robustness of the device under prolonged periods of temperatures exceeding $30^{\circ} \mathrm{C}$ are not yet available.

No DNA contamination events were recorded, despite $50 \%$ of operators not having followed instructions to clean instrument surfaces with bleach and ethanol on a daily basis.

Positive (known smear-positive sputum) and negative controls (Xpert sample reagent buffer) controls were run upon receipt of new lots. No lot-to-lot variability or quality issues with cartridges or sample reagent buffer were observed.

\section{Training needs}

The training duration per laboratory was two days. Operator skill sets prior to this training did not include any experience with molecular tests. Most operators were microscopists and computer knowledge was basic in most cases.

Training was done using a combination of the user manual and PowerPoint slide sets developed by FIND. Training covered a) how to handle the computer and operate the GeneXpert software; $b$ ) the 
sample processing step and associated biosafety; c) the concept of DNA contamination, including the need for cleaning with bleach and disposal of cartridges without re-opening; and d) trouble-shooting.

One-day web-based training was provided by the manufacturer for more experienced operators at two sites and proficiency achieved without any problems. Training for module swap-out, required in case of module replacement or calibration, was also provided by the manufacturer via telephone or internet (web-based).

An anonymous questionnaire survey during the initial FIND validation studies showed that users found the Xpert MTB/RIF assay very simple to operate. User satisfaction with the assay and userindependent read-out was very high.

\section{Waste management}

Cartridge disposal was done according to local guidelines for hazardous materials, identical to sputum containers. Of relevance was the relatively large volume of additional waste generated compared to smear microscopy (cartridges, buffer bottles and single-use pipettes).

\section{Storage of cartridges}

Several of the participating sites could not ensure that room temperature remained below $28^{\circ} \mathrm{C}$ or did not have adequate storage space. Cartridges were therefore stored at supervisory sites with air conditioning or a cool room) and were distributed approximately every two weeks.

\section{Electrical supply and back-up power}

All participating sites reported frequent power outage (seconds to minutes in duration) and small UPS of 400 VA were therefore installed at all sites, sufficient to run for 15-20 minutes during power outages. One site suffering from longer power cuts (lasting hours) used serially connected car batteries to run the GeneXpert instrument and computer in case of power failures, without any apparent adverse effect on instrument or test performance.

\section{Biosafety requirements}

In a series of studies carried out at UMDNJ, the relative biosafety of the Xpert MTB/RIF procedure was compared to routine preparation of slides for direct acid-fast bacilli (AFB) microscopy. Treatment of sputum with a 3:1 ratio of the sample reagent (SR) eliminated $M$. tuberculosis growth in all subsequent cultures while a 2:1 ratio achieved $>6 \log _{10} \mathrm{cfu} / \mathrm{ml}$ killing of $M$. tuberculosis with 15 minutes of exposure.

Aerosolisation studies indicated that no detectable infectious aerosols were generated within the instrument during operation, and inoculating cultures with fluid from inside the cartridges showed $100 \%$ negative cultures.

\subsection{Cost-effectiveness modeling}

Data from three different epidemiological settings (Azerbaijan, India, South Africa) indicated that the use of Xpert MTB-RIF significantly increased TB case-finding (by roughly $30 \%$ ) when used as a replacement or add-on test to microscopy. Use of Xpert MTB/RIF as replacement for conventional culture and DST also significantly increased MDR case-finding (roughly three-fold). The capital and running costs of Xpert MTB/RIF were substantially greater than those of microscopy, though similar to the cost for performing culture and drug susceptibility testing. The total cost per case detected varied considerably by country, but on average the replacement scenario had between $8 \%$ and $11 \%$ higher cost per DALY gained, still within acceptable cost-effectiveness ratios according to WHO criteria. 


\section{Policy recommendations}

The GRADE process confirmed a solid evidence base to support widespread use of Xpert MTB/RIF for detection of TB and rifampicin resistance and resulted in the following main recommendations:

1. Xpert MTB/RIF should be used as the initial diagnostic test in individuals suspected of having MDR-TB or HIV-associated TB. (Strong recommendation)

2. Xpert MTB/RIF may be considered as a follow-on test to microscopy in settings where MDRTB or HIV is of lesser concern, especially in further testing of smear-negative specimens. (Conditional recommendation acknowledging major resource implications)

Remarks:

These recommendations apply to the use of Xpert MTB/RIF in sputum specimens (including pellets from decontaminated specimens). Data on the utility of Xpert MTB/RIF in extrapulmonary specimens are still limited;

These recommendations support the use of one sputum specimen for diagnostic testing, acknowledging that multiple specimens increase the sensitivity of Xpert MTB/RIF but have major resource implications;

These recommendations also apply to children, based on the generalisation of data from adults and acknowledging the limitations of microbiological diagnosis of TB (including MDR-TB) in children;

Access to conventional microscopy, culture and DST is still needed for monitoring of therapy, for prevalence surveys and/or surveillance, and for recovering isolates for drug susceptibility testing other than rifampicin (including second-line anti-TB drugs). 


\section{Implementation considerations}

As with any new technology, a range of implementation issues was identified, without which Xpert MTB/RIF use would not be optimal. These include:

- Positioning: Xpert MTB/RIF is suitable for use at district and sub-district level. Although testing with Xpert MTB/RIF does not require additional laboratory equipment, the sophisticated nature of the device requires care of handling, i.e. stable and uninterrupted electrical supply to avoid interruption of the procedure and subsequent loss of results, security against theft, adequate storage space for the cartridges, dedicated staff to perform testing, and biosafety procedures similar to microscopy;

- Adoption of Xpert MTB/RIF does not eliminate the need for conventional TB microscopy, culture and DST capacity. Microscopy and/or culture remains necessary for monitoring of treatment, as it is unlikely that a test based on DNA detection would be suitable. Xpert MTB/RIF should therefore not be used for monitoring of treatment. In addition, conventional culture and DST are still required to detect resistance to anti-TB drugs other than rifampicin;

- The settings and algorithms for using Xpert MTB/RIF should be guided by country-specific epidemiology (TB, HIV and MDR-TB), available resources, and anticipated cost-effectiveness. Interim diagnostic algorithms are included in the WHO Rapid Implementation of Xpert MTB/RIF, 2011 document.

- Testing costs should also be measured against public health and patient benefits, including direct financial savings associated with decreased diagnostic delays and medical and social benefits and reduced transmission associated with early and appropriate treatment;

- Countries already using molecular line probe assay (LPA) for rapid diagnosis of rifampicin resistance may introduce Xpert MTB/RIF at lower levels of the laboratory service (typically at district- or sub-district level) given that LPA is suitable for high-through-put testing at central/regional laboratory level (http://www.who.int/tb/laboratory/en/);

- The negative predictive value (NPViv) for rifampicin resistance of the Xpert MTB/RIF assay is over $99 \%$ in settings with both low and high prevalence of rifampicin resistance, i.e. a negative result accurately excludes the possibility for rifampicin resistance and no further testing to confirm negative results is required.

- The positive predictive value (PPV $)$ for rifampicin resistance using Xpert MTB/RIF exceeds $90 \%$ in settings or patient groups where the underlying prevalence of rifampicin resistance is greater than $15 \%$. The PPV ranges between $71 \%$ and $84 \%$ where prevalence of rifampicin resistance is between $5 \%$ and $10 \%$, and diminishes further to less than $70 \%$ when the prevalence of underlying rifampicin resistance falls below $5 \%$. In such circumstances, positive Xpert MTB/RIF results should be confirmed by conventional DST or LPA. The PPV of Xpert MTB/RIF can be greatly improved by careful risk assessment in individual patients and targeted Xpert MTB/RIF testing, as outlined in the WHO Rapid Implementation of Xpert MTB/RIF, 2011 document;

- Adoption of Xpert MTB/RIF should be decided by Ministries of Health within the context of national plans for management of TB, MDR-TB and HIV-associated TB, including the development of country-specific screening strategies, timely access to quality-assured first- and second-line anti-tuberculosis drugs, and appropriate care delivery mechanisms;

\footnotetext{
iv NPV for rifampicin resistance: the proportion of diagnosed rifampicin-susceptible cases that are truly susceptible v PPV for rifampicin resistance: the proportion of diagnosed rifampicin-resistant cases that are truly resistant
} 
- Adoption of Xpert MTB/RIF should be phased in within the context of national laboratory strengthening plans, considering that the GeneXpert system may also provide a technology platform for other diagnostic services and reducing costs involved in providing integrated laboratory services;

- As Xpert MTB/RIF only detects resistance to rifampicin, countries with documented or suspected cases of XDR-TB should establish or expand conventional culture and second-line DST capacity for quality-assured testing of second-line drugs, based on current WHO policy guidance;

- Xpert MTB/RIF cartridges and the specimen reagent should be stored at $2-28^{\circ} \mathrm{C}$ as per manufacturer recommendations. The cartridges are quite bulky when packed and require substantial storage space.

- The maximum capacity of a single, four-module GeneXpert instrument is $\mathbf{2 0}$ specimens per day. Busier sites will therefore either need several four-module instruments or larger instruments (16 modules or more), with associated cost and storage implications.

- The manufacturer recommended ambient operating temperature for the GeneXpert instrument is currently limited to a maximum of $30^{\circ} \mathrm{C}$. In settings where the ambient temperature regularly exceeds $30^{\circ} \mathrm{C}$, air conditioning of the room where the assay is being done should be considered;

- The Xpert MTB/RIF cartridges have a shelf life of 18 months, posing a challenge in relatively inaccessible areas with complex customs clearance procedures. Xpert MTB/RIF cartridges are usually shipped with a manufacturer-guaranteed minimum shelf-life of 6-9 months from the date of shipment. Management of inventory based on usage, shelf-life and lead time for delivery of orders is therefore needed;

- The GeneXpert modules require annual calibration which must be performed by a commercial distributor or carried out by swapping old modules for calibrated modules. A detailed commercial sales contract and customer support plan should be negotiated with the supplier, guaranteeing ample and continuous supply of cartridges, customs clearance, maintenance and calibration, repair and replacement;

- Mechanisms for rapid reporting of Xpert MTB/RIF results to clinicians and timely access to appropriate treatment must be established to provide patients with the benefit of an early diagnosis;

- The capital and running costs of Xpert MTB/RIF are substantially greater than those of microscopy, and currently similar to the cost for performing conventional culture and DST. Costs of manufacture (and therefore purchase) are expected to decrease substantially as demand and manufacturing volume increases. FIND has negotiated preferentially pricing of Xpert MTB/RIF for the public health sector in developing countries; more information can be found on http://finddiagnostics.org. 


\section{Research implications}

A series of operational research questions have been identified related to the introduction of Xpert MTB/RIF and its impact on the diagnosis of TB and MDR-TB and patient management While these should not prevent or delay the implementation of Xpert MTB/RIF, priorities for research include:

- Evaluation of the interim diagnostic algorithms in different epidemiological and geographical settings and patient populations;

- Cost-effectiveness and cost-benefit of Xpert MTB/RIF in different programmatic settings;

- Evaluation of Xpert MTB/RIF in extra-pulmonary and paediatric TB;

- Evaluation of the impact on Xpert MTB/RIF in reducing the diagnostic delay and the time for initiation of appropriate treatment;

- Evaluation of the impact of Xpert MTB/RIF on treatment access and treatment outcomes, especially among hard-to-reach populations.

Key operational research questions for Xpert MTB/RIF are outlined in the WHO Rapid Implementation of Xpert MTB/RIF, 2011 document.

\section{Plans for supporting roll-out of Xpert MTB/RIF}

A Global Consultation was called by WHO on 30 November - 2 December 2010 where implementation considerations for roll-out of Xpert MTB/RIF were discussed and broad consensus on the way forward was achieved. Key outcomes of the consultation were agreement on interim diagnostic algorithms, the positioning of Xpert MTB/RIF in risk groups at different levels of health services, and implementation considerations for programmatic roll-out of Xpert MTB/RIF to maximise use and benefits of the technology. Interim diagnostic algorithms initially developed in consultation with the respective Working Groups of the Stop TB Partnership (GLI, MDR-TB, DOTS Expansion and TB/HIV), were discussed in depth and revised during the Global Consultation meeting.

In March 2010, the Rapid Implementation document developed by the WHO Stop TB Department was disseminated widely through e-mail distribution and placed on various websites of stakeholders. The Rapid Implementation document outlines the requirements for systematic roll-out of Xpert MTB/RIF in varying epidemiological and resource settings, with a view towards future large-scale implementation based on programmatic data collected during the roll-out phase. The document contains the interim diagnostic algorithms, recommended patient management approaches, and operational/logistical aspects which need to addressed during Xpert MTB/RIF implementation.

With the cooperation of partners, WHO will provide global coordination of Xpert roll-out to avoid duplication and overlap of efforts. During the roll-out phase a dedicated website will be established to map uptake of Xpert MTB/RIF, communicate operational problems reported from the field and corrective measures taken. Countries and partners embarking on Xpert MTB/RIF roll-out are encouraged to join this effort, use the interim diagnostic algorithms, and contribute to the standardised collection of data. A meeting of Early Implementers will be called by WHO at the end of 2011 to share and review findings. Results and subsequent refinement of testing strategies from the roll-out phase will be used to inform future scale-up of Xpert MTB/RIF at country level. 


\section{References}

1. Schünemann H.J., Oxman A.D., Brozek J., Glasziou P., Jaeschke R., Vist G.E., Williams J.W., Jr., Kunz R., Craig J., Montori V.M., Bossuyt P., Guyatt G.H. Grading quality of evidence and strength of recommendations for diagnostic tests and strategies. BMJ, 2008;336:1106-1110. PMID: 18483053

2. WHO STAG-TB Report 2010. Available at: http://www.who.int/tb/advisory bodies/stag tb report 2010.pdf.

3. Xpert MTB/RIF: Expert Group Meeting Report. WHO, Geneva. September 2010. Available at: http://www.who.int/tb/laboratory/en/

4. WHO Framework for Implementing TB Diagnostics, 2011. Available at: http://www.who.int/tb/laboratory/whopolicy framework mar2011.pdf

5. Rapid Implementation of Xpert MTB/RIF. WHO, Geneva WHO/HTM/TB/2011.2, 2011. Available at: http://www.who.int/tb/laboratory/en/

\section{PUBLISHED MANUSCRIPTS}

1. Banada PP, Sivasubramani SK, Blakemore R, Boehme C, Perkins MD, Fennelly K, et al. Containment of bioaerosol infection risk by the Xpert MTB/RIF assay and its applicability to point-of-care settings. Journal of Clinical Microbiology, 2009. Oct;48(10):3551-7.

2. Blakemore R, Story E, Helb D, Kop J, Banada P, Owens MR, et al. Evaluation of the analytical performance of the Xpert MTB/RIF assay. Journal of Clinical Microbiology, 2009. Jul;48(7):2495-501.

3. Boehme CC, Nabeta P, Hillemann D, Nicol MP, Shenai S, Krapp F, et al. Rapid molecular detection of tuberculosis and rifampin resistance. The New England Journal of Medicine, 2010; Sep;363(11):1005-15.

4. Boehme CC, Nichol MP, Nabeta P, et al. Feasibility, diagnostic accuracy, and effectiveness of decentralised use of the Xpert MTB/RIF test for diagnosis of tuberculosis and multidrug resistance: a multicentre implementation study. Lancet 2011 Published online April 19, 2011 DOI:10.1016/S0140-6736(11)60438-8.

5. Helb D, Jones M, Story E, Boehme C, Wallace E, Ho K, et al. Rapid detection of Mycobacterium tuberculosis and rifampin resistance by use of on-demand, near-patient technology. Journal of Clinical Microbiology 2010. Jan;48(1):229-37.

6. Marlowe EM, Novak-Weekley SM, Cumpio J, et al. Evaluation of the Cepheid Xpert MTB/RIF assay for the direct detection of Mycobacterium tuberculosis complex from respiratory specimens. Journal of Clinical Microbiology 2011. Published online 2 February, 2011 DOI:10.1128/JCM.02214-10.

7. Moure R, et al. Rapid detection of Mycobacterium tuberculosis complex and rifampin resistance in smearnegative clinical samples by use of an integrated real-time PCR method. Journal of Clinical Microbiology. 2011 Mar;49(3):1137-9.

8. Small PM, Pai M. Tuberculosis diagnosis-time for a game change. The New England Journal of Medicine 2010. Sep 9;363(11):1070-1.

9. Theron G, Peter J, et al . Evaluation of the Xpert ${ }^{\circledR}$ MTB/RIF assay for the diagnosis of pulmonary tuberculosis in a high HIV prevalence setting. Am. J. Respir. Crit. Care Med. published ahead of print on April 14, 2011 as doi:10.1164/rccm.201101-00560C .

10. Van Rie A, Page-Shipp L, Scott L, Sanne I, Stevens W. Xpert((R)) MTB/RIF for point-of-care diagnosis of TB in high-HIV burden, resource-limited countries: hype or hope? Expert Review of Molecular Diagnostics 2010. Oct;10(7):937-46.

\section{PUBLISHED POSTERS}

1. Catharina C Boehme, Pamela Nabeta, Girts Skenders, Tatiana Caceres, Lee Christel, David Alland, Martin Jones and Mark D Perkins. First evaluation of Xpert MTB prototype assay for rapid detection of pulmonary TB and MDR TB in Peru and Latvia. IUATLD, Paris, October 2008.

2. S. Naidoo (Lancet Laboratories, South Africa). Evaluation of GeneXpert MTB/RIF assay on pulmonary and extrapulmonary samples in a high throughput routine laboratory. ECCMID, Vienna, April, 2010.

3. S. Naidoo (Lancet Laboratories, South Africa). Evaluation of Xpert MTB/RIF assay on pulmonary samples in a high throughput routine laboratory. Pathvine Congress, Cape Town, August, 2010.

4. P. Ioannidis, D. Papaventsis, S. Nikolaou, S. Karabela, M. Panagi, A. Raftopoulou, E. Konstantinidou, I. Marinou, S. Kanavaki (National Reference Laboratory, Greece) Evaluation of GeneXpert MTB/RIF Assay for 
MTB Detection and Rifampicin resistance in Athens, Greece. $31^{\text {st }}$ Annual ESM Congress, Bled, Slovenia, July 2010.

5. Nataša Fajfar, Urška Bidovec-Stojkovič, Manca Žolnir-Dovč (University Clinic of Respiratory and Allergic Diseases Golnik, Slovenia) Evaluation of GeneXpert MTB/RIF assay for detection of Mycobacterium tuberculosis and rifampicin resistance in a routine laboratory setting in Slovenia. $31^{\text {st }}$ Annual ESM Congress, Bled, Slovenia, July 2010.

6. M.T. Tórtola, L. Nieto, M.G. Codina, N. Martín-Casabona (Microbiologia, Hospital Universitari Vall d’Hebron. Barcelona) Detection of $M$. tuberculosis and rifampicin-resistance using a commercial PCR real time technique in respiratory and extrapulmonary samples. $31^{\text {st }}$ Annual ESM Congress, Bled, Slovenia, July 2010.

7. B. Malbruny, G. Le Marrec, K. Courageux, R. LeClerq, V. Cattoir (Service de Microbiologie, CHU Côte de Nacre, Caen, France). Rapid and Efficient Detection of Mycobacterium tuberculosis by the Cepheid Xpert MTB/RIF Assay, ICAAC, Boston, September 2010.

8. T. Bodmer, and A. Ströhle (University of Berne, Switzerland). Diagnosing pulmonary tuberculosis in a low prevalence setting: the Xpert ${ }^{\circledR}$ MTB/RIF test. ESM, 2010.

9. S. Filippo, Mitchelmore I, Pillai P, Mulla R (Luton and Dunstable Hospital NHS Foundation Trust, UK), Rapid diagnosis of Mycobacterium tuberculosis using Cepheid Xpert ${ }^{\mathrm{TM}}$ MTB/RIF PCR. HPA conference, 2010 
10. GRADE tables 
Table 1. GRADE evidence profile: Xpert MTB/RIF as a replacement test for conventional culture and DST in FIND- coordinated multicentre evaluation and demonstration studies

\begin{tabular}{|c|c|c|c|c|c|c|c|c|}
\hline $\begin{array}{l}\text { No of participants } \\
\text { (studies) }\end{array}$ & $\begin{array}{l}\text { Study } \\
\text { design }\end{array}$ & Limitations & Indirectness & Inconsistency & Imprecision & $\begin{array}{c}\text { Publication } \\
\text { bias }\end{array}$ & $\begin{array}{c}\text { Quality of evidence } \\
\text { (GRADE) }\end{array}$ & Importance \\
\hline \multicolumn{9}{|c|}{ A. Outcome: Diagnostic accuracy for detection of pulmonary TB } \\
\hline \multicolumn{9}{|l|}{ True Positives } \\
\hline $1,275(10)^{A 1}$ & $\begin{array}{l}\text { Cross- } \\
\text { sectional }\end{array}$ & $\underset{A 2}{\text { Serious }}(-1)$ & $\begin{array}{c}\text { No serious } \\
\text { indirectness }^{\mathrm{A3}}\end{array}$ & $\begin{array}{c}\text { No serious } \\
\text { inconsistency }\end{array}$ & $\begin{array}{c}\text { No serious } \\
\text { imprecision }^{\mathrm{A} 5}\end{array}$ & Not likely $^{A 6}$ & $\begin{array}{c}\text { Moderate } \\
\oplus \oplus \oplus \bigcirc\end{array}$ & $\begin{array}{c}\text { Critical } \\
(7-9)\end{array}$ \\
\hline \multicolumn{9}{|l|}{ True Negatives } \\
\hline $2,447(10)^{A 1}$ & $\begin{array}{c}\text { Cross- } \\
\text { sectional }\end{array}$ & $\underset{A 2}{\text { Serious }}(-1)$ & $\begin{array}{c}\text { No serious } \\
\text { indirectness }^{\mathrm{A3}}\end{array}$ & $\begin{array}{c}\text { No serious } \\
\text { inconsistency }\end{array}$ & $\begin{array}{c}\text { No serious } \\
\text { imprecision }^{\text {A5 }}\end{array}$ & Not likely ${ }^{A 6}$ & $\begin{array}{c}\text { Moderate } \\
\oplus \oplus \oplus \bigcirc\end{array}$ & $\begin{array}{c}\text { Critical } \\
(7-9)\end{array}$ \\
\hline \multicolumn{9}{|l|}{ False Positives } \\
\hline $31(10)^{A 1}$ & $\begin{array}{c}\text { Cross- } \\
\text { sectional }\end{array}$ & $\underset{\mathrm{A} 2}{\operatorname{Serious}}(-1)$ & $\begin{array}{c}\text { No serious } \\
\text { indirectness }\end{array}$ & $\begin{array}{c}\text { No serious } \\
\text { inconsistency }{ }^{\mathrm{A4}}\end{array}$ & $\begin{array}{c}\text { No serious } \\
\text { imprecision }^{\mathrm{A} 5}\end{array}$ & Not likely ${ }^{A 6}$ & $\begin{array}{c}\text { Moderate } \\
\oplus \oplus \oplus \bigcirc\end{array}$ & $\begin{array}{c}\text { Critical } \\
(7-9)\end{array}$ \\
\hline \multicolumn{9}{|l|}{ False Negatives } \\
\hline $118(10)^{A 1}$ & $\begin{array}{c}\text { Cross- } \\
\text { sectional }\end{array}$ & $\underset{A 2}{\text { Serious }}(-1)$ & $\begin{array}{c}\text { No serious } \\
\text { indirectness }^{\mathrm{A3}} \\
\end{array}$ & $\begin{array}{c}\text { No serious } \\
\text { inconsistency }\end{array}$ & $\begin{array}{c}\text { No serious } \\
\text { imprecision }^{\text {A5 }}\end{array}$ & Not likely ${ }^{A 6}$ & $\begin{array}{c}\text { Moderate } \\
\oplus \oplus \oplus \bigcirc\end{array}$ & $\begin{array}{c}\text { Critical } \\
(7-9)\end{array}$ \\
\hline \multicolumn{9}{|c|}{ B. Outcome: Diagnostic accuracy for rifampicin resistance detection } \\
\hline \multicolumn{9}{|l|}{ True Positives } \\
\hline $376(10)^{\mathrm{A} 1}$ & $\begin{array}{c}\text { Cross- } \\
\text { sectional }\end{array}$ & $\underset{A 2}{\text { Serious }}(-1)$ & $\begin{array}{c}\text { No serious } \\
\text { indirectness }^{A 3}\end{array}$ & $\begin{array}{c}\text { No serious } \\
\text { inconsistency }{ }^{\mathrm{A4}}\end{array}$ & $\begin{array}{c}\text { No serious } \\
\text { imprecision }^{\text {A5 }}\end{array}$ & Not likely ${ }^{A 6}$ & $\begin{array}{c}\text { Moderate } \\
\oplus \oplus \oplus \bigcirc\end{array}$ & $\begin{array}{c}\text { Critical } \\
(7-9)\end{array}$ \\
\hline \multicolumn{9}{|l|}{ True Negatives } \\
\hline $1,171(10)^{\mathrm{A} 1}$ & $\begin{array}{c}\text { Cross- } \\
\text { sectional }\end{array}$ & $\underset{A 2}{\text { Serious }}(-1)$ & $\begin{array}{c}\text { No serious } \\
\text { indirectness }\end{array}$ & $\begin{array}{c}\text { No serious } \\
\text { inconsistency }\end{array}$ & $\begin{array}{c}\text { No serious } \\
\text { imprecision }^{\text {A5 }}\end{array}$ & Not likely ${ }^{\mathrm{AG}}$ & $\begin{array}{c}\text { Moderate } \\
\oplus \oplus \oplus \bigcirc\end{array}$ & $\begin{array}{c}\text { Critical } \\
(7-9)\end{array}$ \\
\hline \multicolumn{9}{|l|}{ False Positives } \\
\hline $21(10)^{A 1}$ & $\begin{array}{c}\text { Cross- } \\
\text { sectional }\end{array}$ & $\underset{A 2}{\text { Serious }}(-1)$ & $\begin{array}{c}\text { No serious } \\
\text { indirectness }^{A 3}\end{array}$ & $\begin{array}{c}\text { No serious } \\
\text { inconsistency }\end{array}$ & $\begin{array}{c}\text { No serious } \\
\text { imprecision }^{\text {A5 }}\end{array}$ & Not likely ${ }^{A 6}$ & $\begin{array}{c}\text { Moderate } \\
\oplus \oplus \oplus \bigcirc\end{array}$ & $\begin{array}{c}\text { Critical } \\
(7-9)\end{array}$ \\
\hline \multicolumn{9}{|l|}{ False Negatives } \\
\hline $14(10)^{\mathrm{A} 1}$ & $\begin{array}{c}\text { Cross- } \\
\text { sectional }\end{array}$ & $\underset{A 2}{\text { Serious }}(-1)$ & $\begin{array}{c}\text { No serious } \\
\text { indirectness }\end{array}$ & $\begin{array}{c}\text { No serious } \\
\text { inconsistency }{ }^{\mathrm{A4}}\end{array}$ & $\begin{array}{l}\text { No serious } \\
\text { imprecision }{ }^{\text {A5 }}\end{array}$ & Not likely ${ }^{A 6}$ & $\begin{array}{l}\text { Moderate } \\
\oplus \oplus \oplus \bigcirc\end{array}$ & $\begin{array}{c}\text { Critical } \\
(7-9)\end{array}$ \\
\hline
\end{tabular}




\section{Footnotes:}

${ }^{1}$ Quality of evidence was rated as high (no points subtracted), moderate (1 point subtracted), low ( 2 points subtracted), or very low (>2 points subtracted) based on five criteria: study limitations, indirectness of evidence, inconsistency in results across studies, imprecision in summary estimates, and likelihood of publication bias. For each outcome, the quality of evidence was regarded as high since all studies were cross-sectional or cohort studies, prospectively enrolling patients suspected with TB and/or MDR-TB. The evidence rankings were considered to be the same for consideration of true positives, false positives, false negatives, and true negatives.

${ }^{\text {A1 }}$ Sensitivity and specificity of the Xpert MTB/RIF assay for detection of TB were determined using conventional culture as index test in 10 studies coordinated by FIND. These studies also included an assessment of the sensitivity and specificity of the Xpert MTB/RIF system for the detection of rifampicin resistance using conventional DST as the index test. Discordant rifampicin results were resolved by rpoB sequencing.

${ }^{A 2}$ Study limitations were assessed using the QUADAS tool. It was noted that the majority of studies did not enrol children and that the studies were conducted in large urban settings due to the need for quality-assured culture and DST as reference methods. As the intended use of the test is in district and sub-district levels, the quality of evidence was downgraded.

${ }^{\text {A3 }}$ Diagnostic accuracy was considered as a surrogate for patient-important outcomes.

${ }^{A 4}$ No significant heterogeneity among studies was observed, with consistency in sensitivity and specificity estimates reported in different epidemiological settings.

${ }^{A 5}$ Pooled sensitivity derived from the studies had narrow confidence intervals.

${ }^{\text {A6 }}$ Publication bias was considered highly unlikely as the Xpert MTB/RIF is a new assay, produced by a single manufacturer and only available to a small number of investigators. 
Table 2.

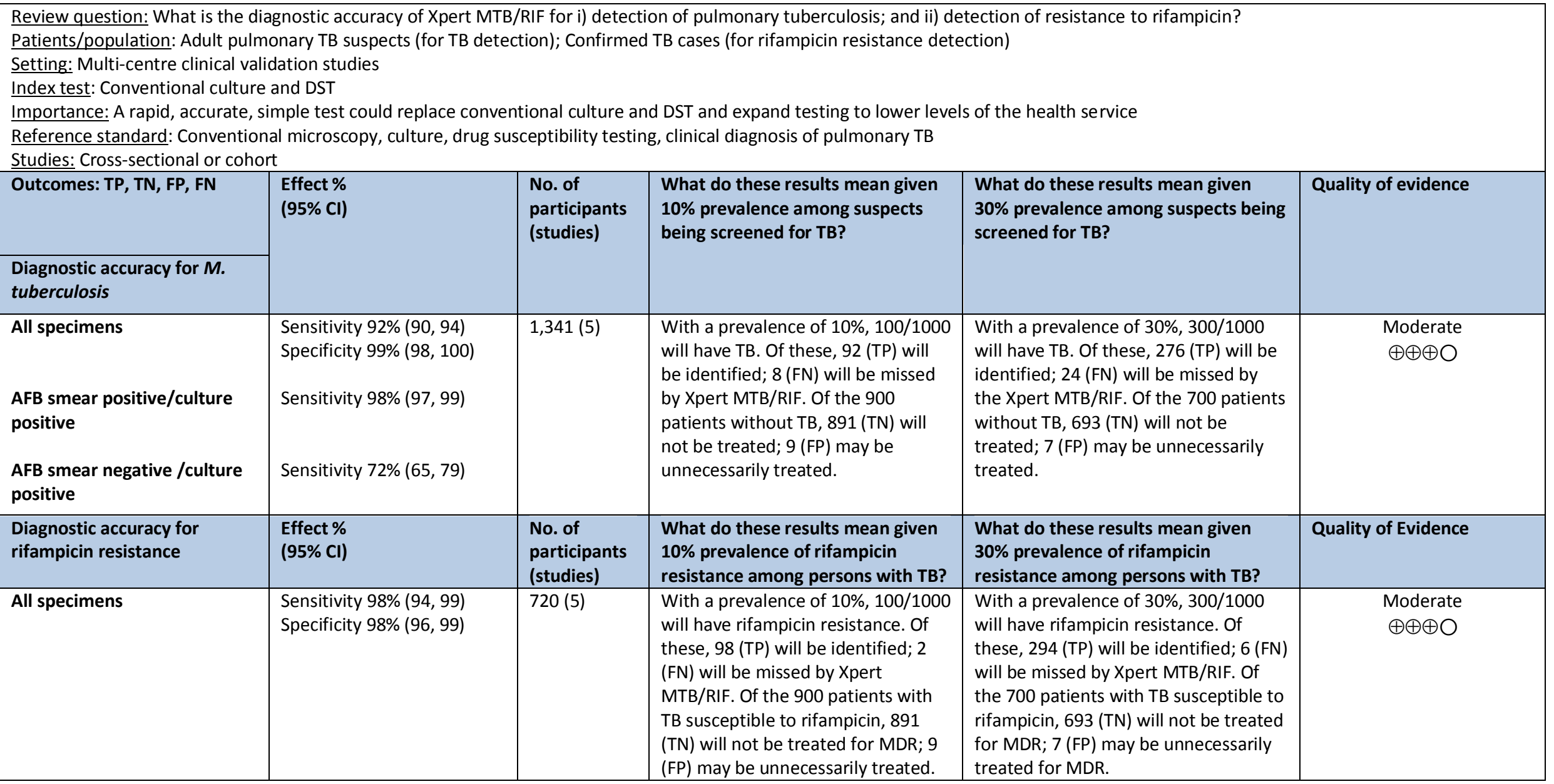




\section{Table 3. GRADE summary of findings - Diagnostic accuracy of the Xpert MTB/RIF assay in multi-centre demonstration studies}

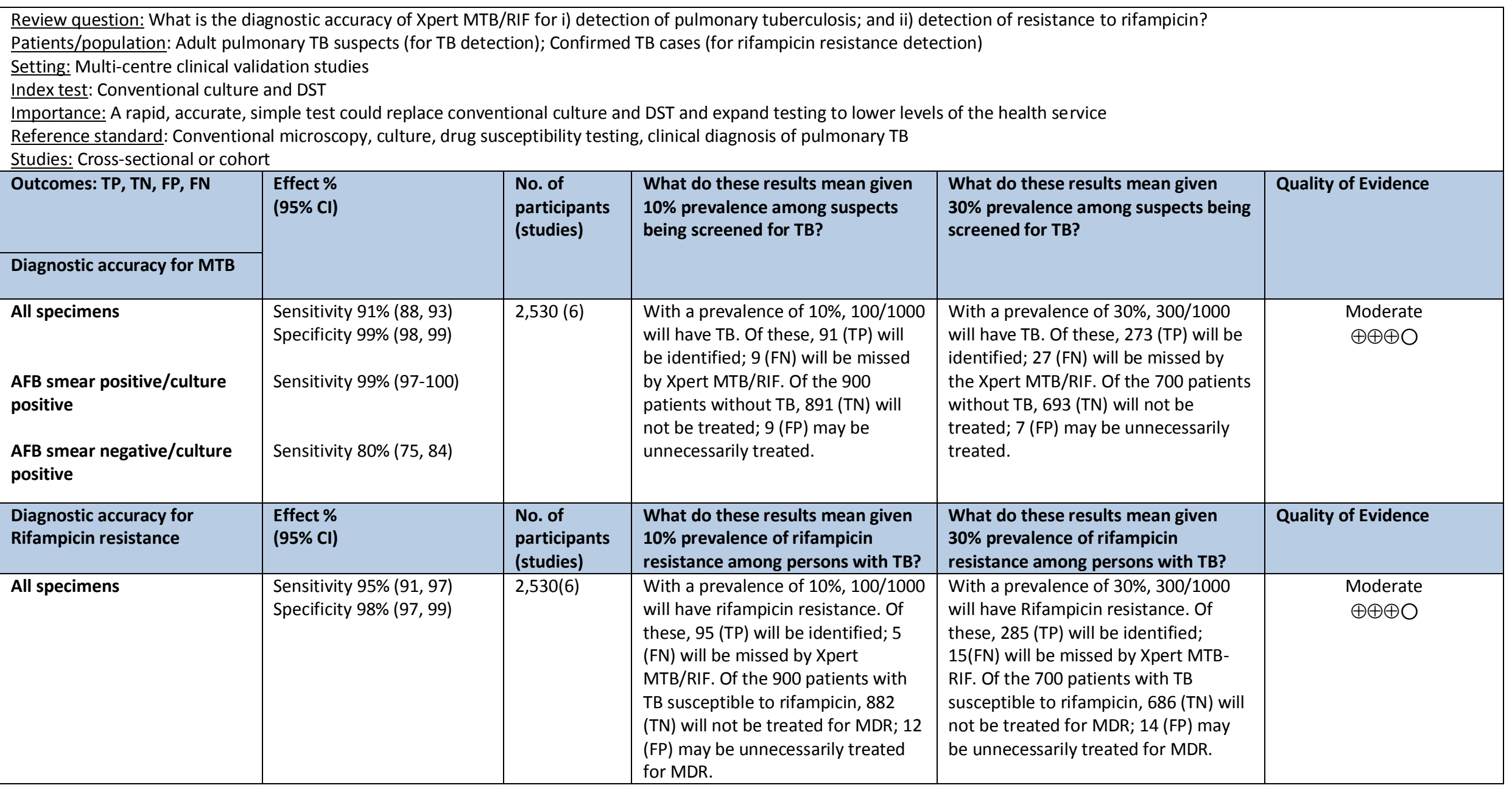




\section{Table 4. GRADE summary of findings - Diagnostic accuracy of the Xpert MTB/RIF assay in single-centre unpublished studies ${ }^{1}$}

\begin{tabular}{|c|c|c|c|c|c|}
\hline \multicolumn{6}{|c|}{$\begin{array}{l}\text { Review question: What is the diagnostic accuracy of Xpert MTB/RIF for i) detection of pulmonary tuberculosis; and ii) detection of resistance to } \\
\text { Patients/population: Adult pulmonary TB suspects (for TB detection); confirmed TB cases (for rifampicin resistance detection) } \\
\text { Setting: Multi-centre clinical validation studies } \\
\text { Index test: Conventional culture and DST } \\
\text { Importance: A rapid, accurate, simple test could replace conventional culture and DST and expand testing to lower levels of the health service } \\
\text { Reference standard: Composite reference standards (L culture, histology/cytology, ADA for CSF and fluids, CT for CSF, follow-up at } 3 \text { months) } \\
\text { Studies: Cross-sectional or cohort }\end{array}$} \\
\hline Outcomes: TP, TN, FP, FN & Crude pooled effect $\%^{1}$ & $\begin{array}{l}\text { No. of } \\
\text { participants } \\
\text { (studies) }\end{array}$ & $\begin{array}{l}\text { What do these results mean given } \\
10 \% \text { prevalence among suspects } \\
\text { being screened for TB? }\end{array}$ & $\begin{array}{l}\text { What do these results mean given } \\
30 \% \text { prevalence among suspects being } \\
\text { screened for TB? }\end{array}$ & Quality of Evidence \\
\hline \multicolumn{6}{|l|}{ Diagnostic accuracy for MTB } \\
\hline $\begin{array}{l}\text { All specimens pulmonary and } \\
\text { extrapulmonary }\end{array}$ & $\begin{array}{l}\text { Sensitivity } 92.5 \% \\
\text { Specificity } 98.0 \%\end{array}$ & $4,373(10)$ & $\begin{array}{l}\text { With a prevalence of } 10 \%, 100 / 1000 \\
\text { will have TB. Of these, } 92 \text { (TP) will } \\
\text { be identified; } 8 \text { (FN) will be missed } \\
\text { by Xpert MTB/RIF. Of the } 900 \\
\text { patients without TB, } 882 \text { (TN) will } \\
\text { not be treated; } 18 \text { (FP) may be } \\
\text { unnecessarily treated. }\end{array}$ & $\begin{array}{l}\text { With a prevalence of } 30 \%, 300 / 1000 \\
\text { will have TB. Of these, } 278 \text { (TP) will be } \\
\text { identified; } 12 \text { (FN) will be missed by the } \\
\text { Xpert MTB/RIF. Of the } 700 \text { patients } \\
\text { without TB, } 686(T N) \text { will not be } \\
\text { treated; } 14 \text { (FP) may be unnecessarily } \\
\text { treated. }\end{array}$ & $\begin{array}{l}\text { Moderate } \\
\oplus \oplus \oplus \bigcirc\end{array}$ \\
\hline $\begin{array}{l}\text { Diagnostic accuracy for } \\
\text { rifampicin resistance }\end{array}$ & Pooled effect \% & $\begin{array}{l}\text { No. of } \\
\text { participants } \\
\text { (studies) }\end{array}$ & $\begin{array}{l}\text { What do these results mean given } \\
10 \% \text { prevalence of rifampicin } \\
\text { resistance among persons with TB? }\end{array}$ & $\begin{array}{l}\text { What do these results mean given } \\
30 \% \text { prevalence of rifampicin } \\
\text { resistance among persons with TB? }\end{array}$ & Quality of Evidence \\
\hline $\begin{array}{l}\text { All specimens (pulmonary and } \\
\text { extrapulmonary) }\end{array}$ & $\begin{array}{l}\text { Sensitivity } 98.6 \% \\
\text { Specificity } 98.8 \%\end{array}$ & $917(3)$ & $\begin{array}{l}\text { With a prevalence of } 10 \%, 100 / 1000 \\
\text { will have rifampicin resistance. Of } \\
\text { these, } 98 \text { (TP) will be identified; } 2 \\
\text { (FN) will be missed by Xpert } \\
\text { MTB/RIF. Of the } 900 \text { patients with } \\
\text { TB susceptible to rifampicin, } 889 \\
\text { (TN) will not be treated for MDR; } 11 \\
\text { (FP) may be unnecessarily treated } \\
\text { for MDR. }\end{array}$ & $\begin{array}{l}\text { With a prevalence of } 30 \%, 300 / 1000 \\
\text { will have rifampicin resistance. Of } \\
\text { these, } 296 \text { (TP) will be identified; } 4(\mathrm{FN}) \\
\text { will be missed by Xpert MTB/RIF. Of } \\
\text { the } 700 \text { patients with TB susceptible to } \\
\text { rifampicin, } 692 \text { (TN) will not be treated; } \\
8 \text { (FP) may be unnecessarily treated for } \\
\text { MDR. }\end{array}$ & $\begin{array}{l}\text { Moderate } \\
\oplus \oplus \oplus \bigcirc\end{array}$ \\
\hline
\end{tabular}

${ }^{1}$ These studies were evaluated individually and crude pooled sensitivity and specificity estimates calculated since meta-analyses was not possible given variability in study design, use of various reference standards and availability of preliminary data only. The quality of evidence was consequently downgraded (on directness) by 1 point. 


\section{Annexes}

Annex 1: Xpert MTB/RIF Expert Group members

Annex 2: WHO staff members

Annex 3: STAG-TB members 


\section{Xpert MTB/RIF Expert Group Members}

ANNEX 1

Dr Richard M. Anthony, PhD

Research Coordinator Tuberculosis

KIT Biomedical Research, Royal Tropical Institute

Meibergdreef 39

1105 AZ Amsterdam

The Netherlands

E-mail:R.Anthony@kit.nl

Area of expertise: Research, Molecular

microbiology, Diagnostics development

Dr Rachel Bauquerez

Public Health Officer

Subgroup on Introducing New Approaches and

Tools (INAT Subgroup)

The Global Fund

E-mail:Rachel.Bauquerez@theglobalfund.org

Area of expertise: Public health, Funding/Donor agency

Dr Catharina Boehme

FIND

Foundation for Innovative New Diagnostics

Avenue de Budé 16

1202 Geneva

Switzerland

E-mail:catharina.boehme@finddiagnostics.org

Area of expertise: TB, Research, Diagnostics

development, Developing country experience

Dr Jeremiah Muhwa Chakaya

Chief Research Officer

Kenya Medical Research Institute

Center for Respiratory Diseases Research

00100 Nairobi

Kenya

E-mail: chakaya@materkenya.com;

chakaya.jm@gmail.com

Area of expertise: TB, Research, Clinical, Public health, Developing country experience, End-user (National TB Control Programme)

Dr Daniela Maria Cirillo, MD PhD

Head, Emerging Bacterial Pathogens Unit

San Raffaele Scientific Institute

Via Olgettina 58

20132 Milan Italy

E-mail: cirillo.daniela@hsr.it

Area of expertise: TB, Research, Clinical,

Microbiology, Developing country experience
Dr Frank Cobelens

Senior Epidemiologist

Research Unit KNCV

Parkstraat 17, Postbus 146

2514 CC The Hague

Netherlands

E-mail: cobelensf@kncvtbc.n

Area of expertise: TB, Research, Epidemiology,

Developing country experience

Dr Gerrit Coetzee

Head

National Tuberculosis Reference Laboratory

National Health Laboratory Service

P.O. Box 1038

Cnr Hospital De Karte Street

Braamfontein

2000 - Johannesburg

South Africa

E-mail: dcoetzee@phfm.uct.ac.za

Area of expertise: TB, Clinical, Microbiology,

Developing country experience

Dr Saidi M. Egwaga

Programme Manager

Ministry of Health and Social Welfare

National TB and Leprosy Programme

P.O. Box 9083

Dar Es Salaam

United Republic of Tanzania

E-mail: tantci@intafrica.com

Area of expertise: End-user (National TB Control

Programme), Developing country experience

Ms Christy Hanson

US Agency for International Development (USAID)

USAID/BGH/HIDN/ID

3.7.23, 3rd Floor

Ronald Reagan Building

20523-5900 - Washington, DC

United States of America

E-mail: chanson@usaid.gov

Area of expertise: Public health, Funding/Donor

agency, Developing country experience

Dr Doris Hillemann

National Reference Laboratory for Mycobacteria

Research Center Borstel

Parkallee 18

D-23845 Borstel

Germany

e-mail: dhillem@fz-borstel.de

Area of expertise: TB, Research, Clinical,

Microbiology, Molecular Microbiology, Developing

country experience 
Dr Aamir Khan

Director

MDR-TB Control Program

The Indus Hospital

Korangi Crossing

75190 Karachi

Pakistan

E-mail: aamir.khan@irdresearch.org

Area of expertise: TB, Clinical, MDR-TB, Developing

country experience

Dr Ruth McNerney

Department of Infectious and Tropical

Diseases

Room 358, London School of Hygiene \& Tropical

Medicine

Keppel Street, London WC1E 7HT

United Kingdom

E-mail: Ruth.Mcnerney@Ishtm.ac.uk

Area of expertise: TB, Research, Diagnostics

development, Developing country experience

Dr Rick O’Brien

Head of Product Evaluation

Foundation for New Innovative New Diagnostics

(FIND)

Avenue de Budé 16

1202 Geneva

Switzerland

E-mail: rick.obrien@finddiagnostics.org

Area of expertise: TB, Research, Diagnostics

development, Developing country experience

Dr Giorgio Roscigno

CEO, FIND

Foundation for Innovative New Diagnostics

Avenue de Budé 16

1202 Geneva

Switzerland

E-mail: Giorgio.roscigno@finddiagnostics.org

Area of expertise: TB, Research, Diagnostics

development, Developing country experience

Dr Holger Schünemann

Chair, Department of Clinical Epidemiology \&

Biostatistics

Department of Clinical Epidemiology \& Biostatistics

McMaster University Health Sciences Centre,

Room 2C10B

1200 Main Street West

Hamilton, ON L8N 3Z5, Canada

E-mail: schuneh@mcmaster.ca

Area of expertise: GRADE methodologist,

Epidemiology
Dr Thomas M. Shinnick

Associate Director for Global Laboratory Activities Centres for Disease Control and Prevention 1600 Clifton Road

MS-G35, NE

30333 Atlanta,GA

United States of America

E-mail: tms1@cdc.gov

Area of expertise: TB, Microbiology, Molecular microbiology, Research

Mr Javid Syed

TB/HIV Project Director

Community Rep.

Treatment Action Group (TAG)

611 Broadway, Suite 308

NY 10012 - New York, New York

United States of America

E-mail: javid.syed@treatmentactiongroup.org

Area of expertise: Patient/Community

representative, Developing country experience 
ANNEX 2

WHO staff members

\section{WHO-HQ}

Léopold Blanc

E-mail: blancl@who.int

Katherine Floyd

E-mail: floydk@who.int

Haileyesus Getahun

E-mail: getahunh@who.int

Christopher Gilpin

E-mail: gilpinc@who.int

Jean Iragena

E-mail: iragenaj@who.int

Wieslaw Jakubowiak

E-mail: jakubowiakw@who.int

Ernesto Jaramillo

E-mail: jaramilloe@who.int

Christian Lienhardt

E-mail: lienhardtc@who.int

Fuad Mirzayev

E-mail: mirzayevf@who.int

Paul Nunn

E-mail: nunnp@who.int

Mario Raviglione

E-mail: raviglionem@who.int

Diana Weil

E-mail: weild@who.int

Karin Weyer

E-mail: weyerk@who.int

Matteo Zignol

E-mail: zignolm@who.int

\section{WHO-Regions}

Dr Akihiro Seita, EMRO

E-mail: seitaa@emro.who.int

Dr Ranjani Ramachandran, SEARO

E-mail: ramachandranr@searo.who.int

\section{WHO-TDR}

Dr Andrew Ramsay

E-mail: ramsaya@who.int

Dr Véronique Vincent

E-mail: vincentv@who.int 
ANNEX 3

STAG-TB members

\section{Dr Salah Al Awaidy}

\section{Director}

Department of Communicable Disease

Surveillance \& Control

DGHA, HQ, Ministry of Health

PO Box 393

Muscat 113

Oman

\section{Dr Kenneth Castro}

Director, Division of TB Elimination

Centers for Disease Control and Prevention

1600 Clifton Road, NE, MS E-10

Atlanta, GA 30333

USA

Dr Jeremiah Muhwa Chakaya

Chief Research Officer,

Centre for Respiratory Diseases Research

Kenya Medical Research Institute

47855

00100 - Nairobi

KENYA

\section{Ms Lucy Chesire}

TB Advocacy Adviser

Kenya AIDS NGOs Consortium

KANCO

Chaka Road off Argwings Kodhek Road, P.O. Box 6986600400 Nairobi

KENYA

\section{Dr Elizabeth Corbett}

Reader in Infectious and Tropical Diseases London School of Tropical Medicine \& Hygiene and MLW Research Programme

PO Box 30096 Chichiri, Blantyre 3,Malawi

\section{Charles L. Daley}

Head

Division of Mycobacterial and Respiratory

Infections

National Jewish Medical and Research Center

5301 Nassau Circle E

Englewood, CO 80113

ETATS-UNIS D'AMÉRIQUE

\section{Dr Pam Das}

Executive Director

The Lancet

32 Jamestown Road

London NW1 7BY

Royaume-Uni

\section{Professor Francis Drobniewski}

Director, Health Protection Agency National

Mycobacterium Reference Unit

Institute for Cell and Molecular Sciences,

Barts and the London School of Medicine

Clinical Sciences Research Center

2 Newark Street

London E1 2AT

United Kingdom

\section{Dr Wafaa El-Sadr}

CIDER

Mailman School of Public Health

Columbia University

506 Lenox Avenue Room 3101A

New York NY 10037

USA

\section{Dr Paula I. Fujiwara}

Senior Technical Advisor

International Union Against Tuberculosis

and Lung Disease (The Union)

68 Blvd St. Michel

75006 Paris

France

\section{Dr Yuthichai Kasetjaroen}

Director

Bureau of Tuberculosis

Ministry of Health

Thailand

\section{Prof Vladimir Malakhov}

National Center for External Quality

Assessment in Laboratory Testing

of Russian Federation

10, Petroverigsky pereulok,

101431, Moscow

Russia

\section{Dr Mao Tang Eang}

Advisor to the Minister of Health

Director, National Center for Tuberculosis and

Leprosy Control

Ministry of Health

Cambodia 


\section{Dr Giovanni Battista Migliori}

Director

WHO Collaborating Centre for Tuberculosis and Lung Diseases

Fondazione Salvatore Maugeri, IRCCS

Via Roncaccio 16

21049 - Tradate, VA

ITALIE

\section{Dr Megan Murray}

Associate Professor of Epidemiology

Department of Epidemiology, Kresge 802

677 Huntington Avenue

Boston, MA 02115

USA

\section{Dr Yogan Pillay}

Deputy Director General

Strategic Health Programmes

Department of Health

Private Bag X828

Pretoria, 0001 Pretoria 0001

South Africa

\section{Dr Minghui Ren}

Director-General

Department of International Cooperation Ministry of Health, China

1 Xizhimenwai Nanlu

Beijing 100044

China

\section{Dr Rajendra Shukla}

Joint Secretary

Ministry of Health \& Family Welfare

Room\&nbsp;\# 147, Wing A

Nirman Bhawan, New Delhi 110008

India

\section{Dr Pedro Guillermo Suarez}

TB \& TB-HIV/AIDS

Center for Health Services

Management Sciences for Health

4301 North Fairfax Drive - Suite 400

Arlington, VA 22203

USA

\section{Dr Marieke van der Werf}

Head, Unit Research, Senior Epidemiologist

KNCV Tuberculosis Foundation

Parkstraat 17

2514 JD The Hague

The Netherlands

Dr Rosalind G. Vianzon

National TB Programme Manager

National Center for Disease Control and Prevention Department of Health

4th Floor, Building 13

San Lazaro Compound, Santa Cruz

Manila

Philippines

Dr Tido Von Schön-Angerer

Executive Director

Campaign for Access to Essential Medicines

Médecins Sans Frontières

CP 116

78 Rue de Lausanne

1211 - Geneva 21 


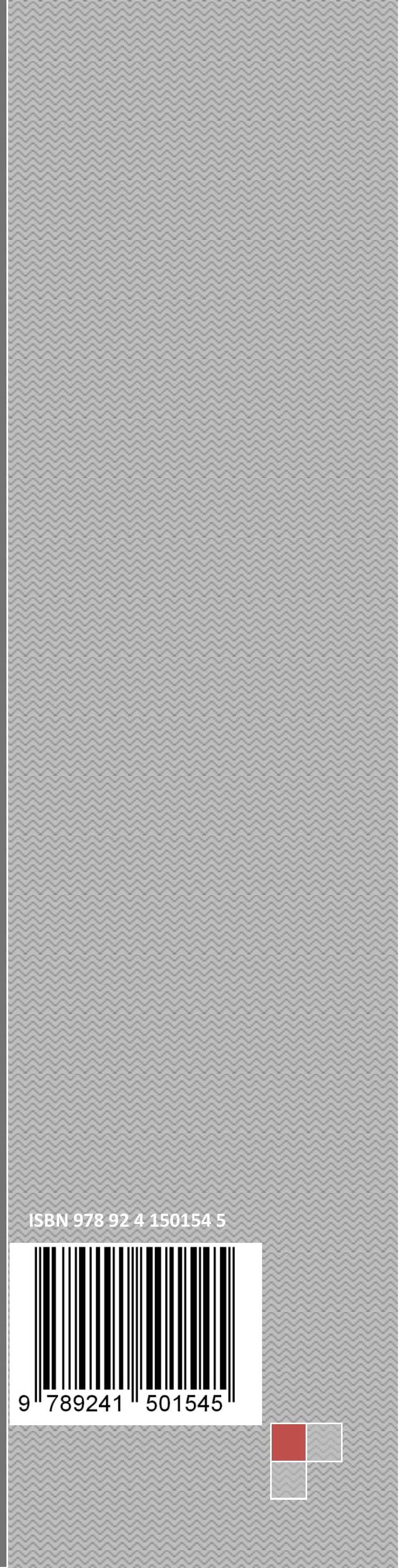

